\title{
FRACTURE OF THE PELVIS GOMPLICATED BY ISCHAEMIA OF THE LOWER LIMB
}

\author{
Report of a Case \\ J. N. Wilson, Oswestry, England \\ From the Robert Jones and Agnes Hunt Orthopaedic Hospital, Osüestry
}

A quarryman aged thirty-seren years was crushed between two wagons in May 1951. When admitted to hospital he complained of pain in the pelvis, with weakness and numbness of the right lower limb. He was found to have sustained a fracture of both pubic rami on the right side with separation of the symphysis and subluxation of the right sacroiliac joint (Fig. 1). His general condition was rather worse than one would expect from a fracture of the pelvis alone, but there were no signs of damage to bladder or urethra, nor of any other

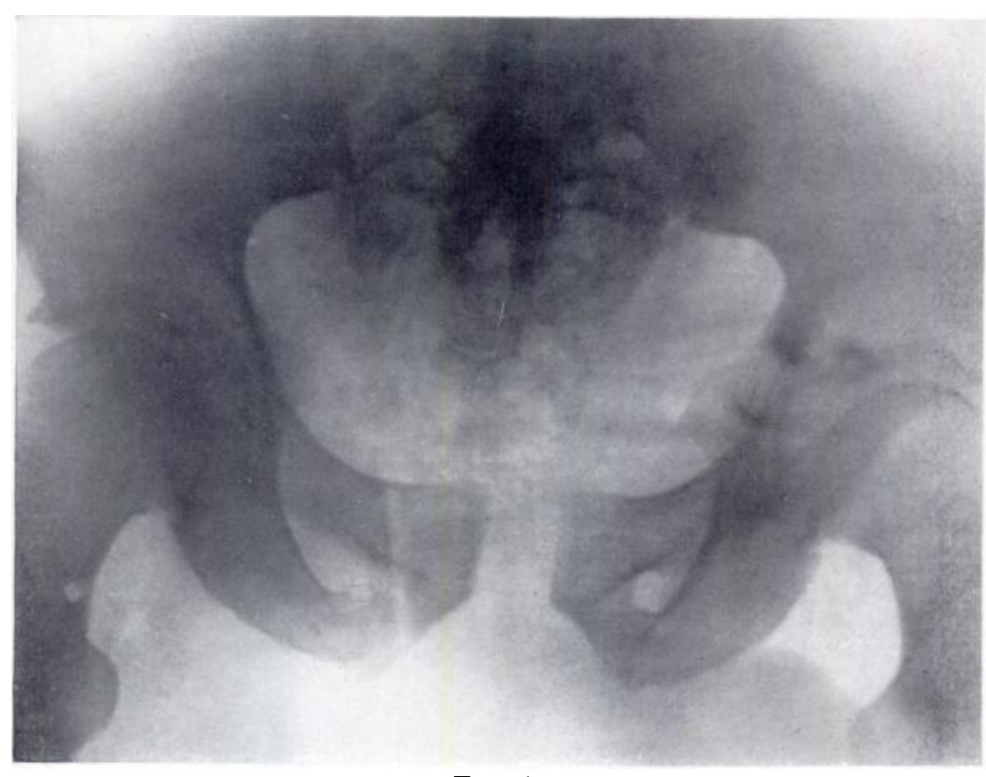

FIG. 1

Radiograph showing separation of the symphysis pubis and fracture of the inferior ramus of the pubis on the right side. The fracture of the superior pubic ramus, found at necropsy, is not discernible.

severe injury. He had, however, sustained considerable local bruising, and it was thought that a large swelling in the right groin was probably a retroperitoneal haematoma. Sensation in the right lower limb was impaired, and there was general weakness in all muscle groups. There was no suggestion at that time of any circulatory disturbance.

His condition improved after admission and he was put up in a pelvic sling in an attempt to correct the separation of the symphysis pubis. About twelve hours later his general condition deteriorated fairly suddenly. The blood pressure dropped to 90 millimetres $\mathrm{Hg}$. (systolic), and it was thought that further bleeding must have occurred into a retroperitoneal haematoma. Two pints of blood were given, with good effect, and the pelvic sling was 
tightened. He soon complained, however, of pain and numbness of the whole of the right lower limb.

On examination, there was found to be no voluntary movement in the limb, and there was complete loss of sensation up to the groin; the ankle was pale and without apparent circulation, and the pulses were impalpable from the femoral downwards. The muscles of the thigh and calf were sharply outlined as they stood out in spasm, the foot being held in rigid equinus. A diagnosis of ischaemia of the right lower limb, probably due to spasm of the femoral artery associated with pressure on the main vessels by an increasing retroperitoneal haematoma, was made. It was thought that the only hope of preserving the limb was to explore the vessels and release the haematoma. But the patient's condition never recovered sufficiently to justify operation, and he died about twelve hours later.

Post-mortem findings - The cause of death was found to be cerebral fat embolism. A large extraperitoneal haematoma was found on the right side extending down under the inguinal ligament, and lying over the femoral vessels. The femoral artery was narrowed in the section overlying the pubic ramus and contained ante-mortem clot in the area of constriction (Fig. 2). The damaged ressel was in immediate relation to a linear fracture of the superior ramus of the pubis, one edge of which impinged against the artery; this edge became more obvious on moving the pelvis. This fracture was not discernible in the radiograph of the pelvis. The muscles of the thigh and calf were pale in comparison with the other side, and the macroscopic appearance was one of ischaemia.

Comment-This case illustrates three points in relation to

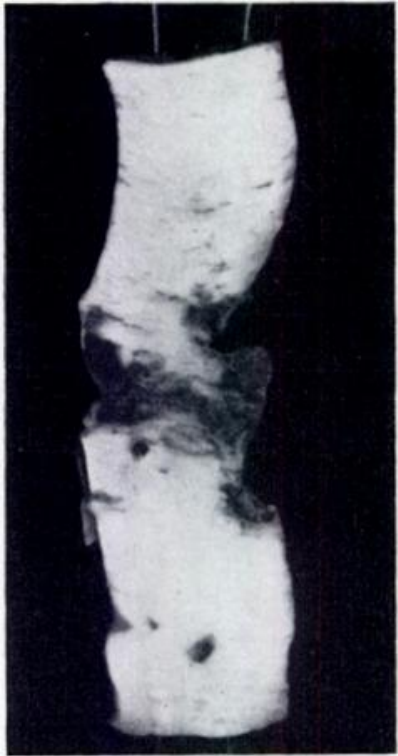

FIG. 2

Part of the femoral artery overlying the right superior pubic ramus. The damaged part of the vessel is shown clearly in the centre of the specimen. femoral artery damage in pelvic injuries. Firstly, how a fracture without evident displacement may damage the artery because of its proximity to the bone at this level. Secondly, the importance of the large retroperitoneal haematoma which may produce local pressure and increase the effect of any spasm already present. Thirdly, it is possible that tightening the pelvic sling may have caused some alteration in the fracture of the pubic ramus, for it was observed that the main symptoms of ischaemia began after the sling had been tightened.

I am indebted to Mr F. G. Allan for permission to publish this case.

VGL. 34 B, NO. 1, FEBRLARY 1952

$\mathrm{EI}_{(4)}$ 